\title{
Current paradigm of the 18-kDa translocator protein (TSPO) as a molecular target for PET imaging in neuroinflammation and neurodegenerative diseases
}

\author{
Alex Sik Chung Ching • Bertrand Kuhnast • \\ Annelaure Damont • Dirk Roeda • Bertrand Tavitian • \\ Frédéric Dollé
}

Received: 4 May 2011 /Revised: 18 June 2011 /Accepted: 9 September 2011 /Published online: 16 October 2011

(C) European Society of Radiology 2011

\begin{abstract}
Neuroinflammation is a process characterised by drastic changes in microglial morphology and by marked upregulation of the $18-\mathrm{kDa}$ translocator protein (TSPO) on the mitochondria. The continual increase in incidence of neuroinflammation and neurodegenerative diseases poses a major health issue in many countries, requiring more innovative diagnostic and monitoring tools. TSPO expression may constitute a biomarker for brain inflammation that could be monitored by using TSPO tracers as neuroimaging agents. From medical imaging perspectives, this review focuses on the current concepts related to the TSPO, and discusses briefly on the status of its PET imaging related to neuroinflammation and neurodegenerative diseases in humans.
\end{abstract}

Keyword TSPO - Translocator protein .

Neuroinflammation · Neurodegenerative diseases .

Microglial activation $\cdot$ PET ligands

\section{Introduction}

Medical imaging-from morphome to molecules

The spectrum of medical imaging extends from morphology, over function, to molecules. X-ray based imaging modalities [traditional X-ray and computed tomography (CT)] primarily deal with anatomy and patterns, whereas radionuclide imaging, including positron emission tomog-

A. S. C. Ching $(\bowtie) \cdot$ B. Kuhnast $\cdot$ A. Damont $\cdot$ D. Roeda

B. Tavitian $\cdot$ F. Dollé

CEA, I2BM, Service Hospitalier Frédéric Joliot,

4 place du Général Leclerc,

91401 Orsay, France

e-mail: chingsc@hotmail.com raphy (PET) and single photon emission computed tomography (SPECT), assess function and physiology [1] (Fig. 1). From the medical viewpoint, clinical molecular imaging can be broadly defined as the in vivo non-invasive visualisation, characterisation and measurement of disease processes at the molecular or cellular level using specific imaging probes [2]. It sets forth to detect the biochemical changes underlying diseases rather than to image the macroscopic structural results of pathological processes.

The current trend of new medical imaging scanner design and development is based on multimodel and multiparametric approaches. Indeed PET and its hybrid imaging modalities $\mathrm{PET} / \mathrm{CT}$, and before long magnetic resonance imaging (MRI)/ PET, are changing the way we practice medicine. To date, $\mathrm{PET} / \mathrm{CT}$ is currently a diagnostic imaging technique used in many hospitals worldwide to provide services for patients, especially in the fields of oncology, neuroscience and cardiology [3]. In spite of the recent advances of molecular and imaging techniques, there is still a gap between basic biomedical research and its clinical applications. Nowadays clinical PET tracer still mainly refers to the commercially available 2-deoxy-2-[ $\left.{ }^{18} \mathrm{~F}\right]$ fluoro-D-glucose $\left(\left[{ }^{18} \mathrm{~F}\right] \mathrm{FDG}\right)$. The impact of PET and its hybrid systems to medicine is limited by the worldwide shortage of radiochemistry facilities to produce novel PET tracers to detect various pathophysiological mechanisms or to specify diagnosis or even theranostics of neurodegenerative diseases.

With the launching of new hybrid imaging systems like MRI/PET, radiologists will expect to play an even more active role in directing development of the rapidly evolving field of medical imaging. Although not all practicing radiologists will engage in the field of research and sciences, it is worthwhile that they keep abreast of recent development of PET tracers in the areas as diverse as oncology, cardiology, metabolic 


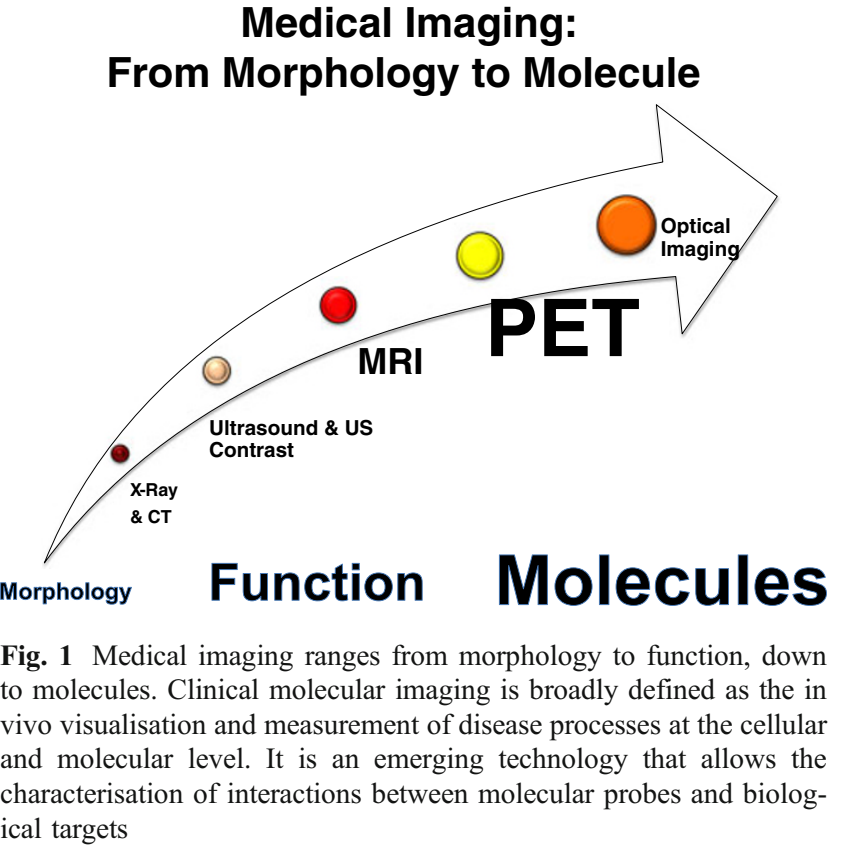

diseases and neurology. In this tutorial article, we review the current concepts related to the pathophysiology of neuroinflammation and neurodegenerative diseases and give a glance on the status of PET imaging of $18-\mathrm{kDa}$ translocator protein (TSPO) tracers in neuroinflammation of the living brain, with the aim of providing a basis for future discussions on the development of TSPO tracers for the diagnosis and therapy of neurodegenerative diseases.

\section{PET is intrinsically quantitative for 3D neuroreceptor imaging}

PET is an imaging technique in which molecular tracers labelled with positron emitters are injected into the human body to track biochemical and physiological processes [3]. Imaging agents used for PET are radioactive probes emitting positively charged anti-electrons (positrons). The positron travels a short distance (less than a millimetre) before it annihilates with any electron to give two photons of $511 \mathrm{keV}$ travelling under a mutual angle of $180^{\circ}$. It is this pair of coincident high-energy photons that underlies the PET principle allowing in vivo quantitative threedimensional (3D) imaging through coincident detection using a gantry of multiple detectors around the patient (Fig. 2). The genuine strength of this powerful molecular imaging modality is its picomolar sensitivity and the possibility of quantitative imaging of in vivo binding to the specific neuroreceptors like TSPO, dopamine receptors and serotonin receptors. PET allows a quantitative 3D reconstruction of the in vivo radioactivity levels of the

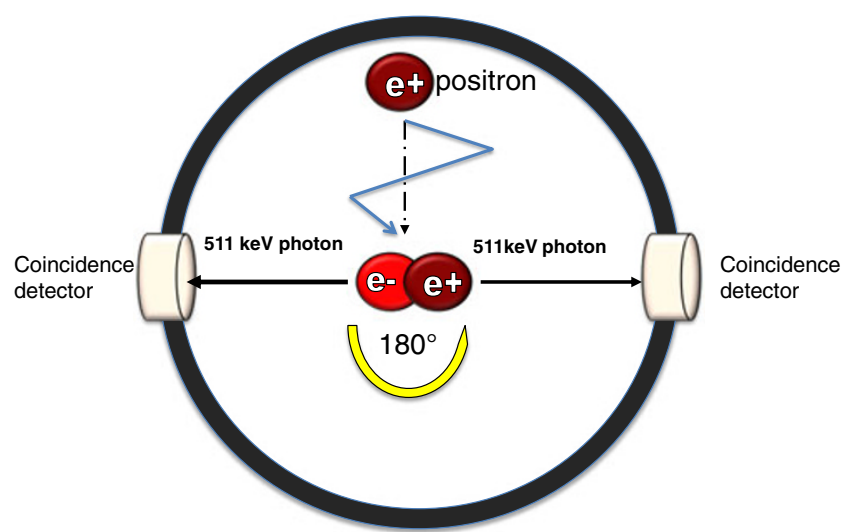

Fig. 2 PET principle: imaging agents used for PET are radioactive probes emitting positively charged anti-electrons (positrons). The positron travels a short distance $(\sim 1-3 \mathrm{~mm})$ before it annihilates with any electron to give two photons of $511 \mathrm{keV}$ travelling under a mutual angle of $180^{\circ}$. It is this pair of coincident high energy photons that underly the PET principle allowing in vivo quantitative 3D imaging through coincident detection using a gantry of multiple detectors around the patient

isotope administrated to a patient [4] (Table 1). It can deliver quantitative measures of biochemical parameters such as the TSPO density in living brain. In vivo assessment of tracer binding to a receptor site is based on the principles of receptor pharmacology [5]. The success of in vivo receptor measurements is predetermined by the understanding of the underlying biological system and by applying the optimal pharmacological model. When done properly, neuroreceptor PET studies can provide useful contributions to clinical studies and also for drug discovery and development.

\section{Brain as an "immune privileged" organ: to be or not to be?}

Neuroinflammation denotes central nervous system (CNS)specific, inflammation-like glial responses that do not reproduce the classic characteristics of inflammation in the periphery but which may engender neurodegenerative events [6]. A neurodegenerative disease is defined as a progressive irreversible deterioration of the motor, sensory or cognitive functions associated with neuronal loss. Traditional concepts have tended to focus attention on pure neurodegenerative or inflammatory mechanisms, neglecting an integrated perspective. These two previously distinct areas of neuroscience are in fact highly related. Since 1873 the brain was classified as an "immune privileged" organ because it seemed to be not affecting by immune responses or inflammation. The organ does not reproduce the classic characteristics of inflammation as in other parts of body. However, there is a recent shift in the fundamental concept 
Table 1 Comparison of radionuclide imaging and MRI as molecular imaging systems [28]

\begin{tabular}{lllll}
\hline Imaging modality & Physiological parameter & Sensitivity & Spatial resolution (mm) & $\begin{array}{l}\text { Minimal amount of tracer } \\
\text { (or contrast agent) used }\end{array}$ \\
\hline PET & Biochemical & $\mathrm{pM}$ & $1-5$ & $\mathrm{ng}$ \\
SPECT & Biochemical & $\mathrm{pM}$ & $1-10$ & $\mathrm{ng}$ \\
MRI & Proton density & $\mu \mathrm{M}$ & $0.1-1^{\mathrm{a}}$ & $\mu \mathrm{mg}$ to mg \\
\hline
\end{tabular}

$p$ pico $\left(10^{-12}\right), \mu$ micro $\left(10^{-6}\right), n$ nano $\left(10^{-9}\right)$

${ }^{\mathrm{a}} 1-3 \mathrm{~mm}$ for fMRI and $5-10 \mathrm{~mm}$ for MRS

as more evidence has suggested the importance of immunerelated inflammatory reactions in the brain [7]. It indicates a more active role of neuroinflammation in the pathophysiology of progressive neurodegenerative disorders. The responses and actions of microglial cells and astrocytes in the brain have an inflammation-like character. These cells play major roles in neuroinflammation and neurodegenerative diseases and they are involved in many complex cellular responses [8]. Many pathological events can trigger neuroinflammation and the primary insult can be infective, degenerative, metabolic or ischaemic. For example, acute inflammation is associated with trauma, stroke and encephalitis, whereas multiple sclerosis (MS), Alzheimer's disease (AD), Parkinson's disease (PD), Huntington's disease (HD), and amyotrophic lateral sclerosis are some of those conditions involving chronic inflammation. Inflammation has been identified as an important factor in primary neurodegenerative disorders. In the past, the associated inflammatory glial responses in these diseases had often been regarded as non-specific "reactive gliosis" that deserved no further significance. This viewpoint might be related to insensitivity of the conventional anatomical imaging modalities, including MRI, to grey matter changes such as plaques, gliosis or subtle atrophy. However, several researchers have discovered that primary neurodegenerative disorders and neuroinflammation are intermingled by immune responses [7, 9] (Fig. 3). Collateral neuronal destruction and myelin damage can occur in the course of neuroinflammation and neurodegeneration [7]. For example, the decline of $\mathrm{N}$ acetyl-aspartate (NAA) levels on MR spectroscopy has been shown to be an indicator for neuronal damage in neurodegenerative diseases such as MS [10]. In the light of these findings, both purely inflammatory and neurodegenerative pathologies are controlled and regulated by the common molecular mechanism with collateral neuronal damage [7]. Initially divergent events such as ischaemia, peroxysomal dysfunction or amyloid beta protein $(A \beta)$ deposition clearly initiate involvement of the immune system, which in turn interacts with the nervous system and ultimately sets the pace of progressive tissue damage.

\section{Microglial activation fans the flame in neuroinflammation}

Microglia are the critical convergence point for the many diverse triggers in orchestrating the activity of other immune cells in the brain [6]. These cells are derived from the monocytic lineage display high sensitivity to different types of CNS injury [11]. During neuroinflammation, microglia undergo drastic changes in their morphology, migrate towards the lesion site, proliferate, and produce neurotoxic factors such as proinflammatory cytokines and reactive oxygen species. Stroke, hypoxia, and trauma compromise neuronal survival and indirectly trigger neuroinflammation as microglia become activated in response to insult in an attempt to limit further injury. A mild autoimmune reaction with microglial activation can have neuroprotective function after CNS injury [9]. An acute insult may trigger oxidative and nitrosative stress, it is typically short lived and unlikely to be detrimental to long-term neuronal survival. On the contrary, chronic neuroinflammation is a longstanding and often selfperpetuating response that persists long after an initial injury or insult. It includes not only longstanding activation of microglia but also subsequent release of inflammatory factors. In chronic inflammation, immune response can be full-blown, including the activation of microglia, and damage the brain tissues by autoimmunogens [12]. That means activated microglia can be either friends or foes to neighbouring neurons. It is not yet clear how to manipulate them to minimise their damaging effects.

\section{Overexpressed TSPO on the mitochondral membrane}

TSPO, formerly known as peripheral benzodiapzepine receptor (PBR), is a ubiquitous high affinity cholesterol transporter found primarily in the outer mitochondrial membrane of steroid-synthesising cells. To a lesser extent, the protein is also found in the cell nucleus and plasma membrane. The new name was adopted in 2006 in view of 
Fig. 3 Collateral neuronal damage is clearly inherent to primary neuroinflammatory diseases, and neuroinflammation is a likely consequence of primary neurodegeneration. a In primary degenerative diseases like $\mathrm{AD}$ and in stroke, a non-immunemediated CNS injury is initiated. Immune response cells, predominantly local microglia, marcophages and lymphocytes, are involved in the degenerative processes and elicit a secondary inflammatory reaction, which sets the pace of progressive neuronal damage. Some of these mechanisms might also occur at certain stages of MS. b In primary inflammatory CNS disease such as MS, activated immune cells - namely, microglia, macrophages and lymphocytes - attack a common target antigen of the brain, typically part of the myelin sheath. Demyelination, axonal transection and neuronal death can occur during the immune response. The loss of myelin might result in secondary axonal transection (outside-in damage) or, vice versa, death of an axon can lead to secondary demyelination (inside-out damage).

Some of these sequences constitute the pathogenesis of diseases like AD
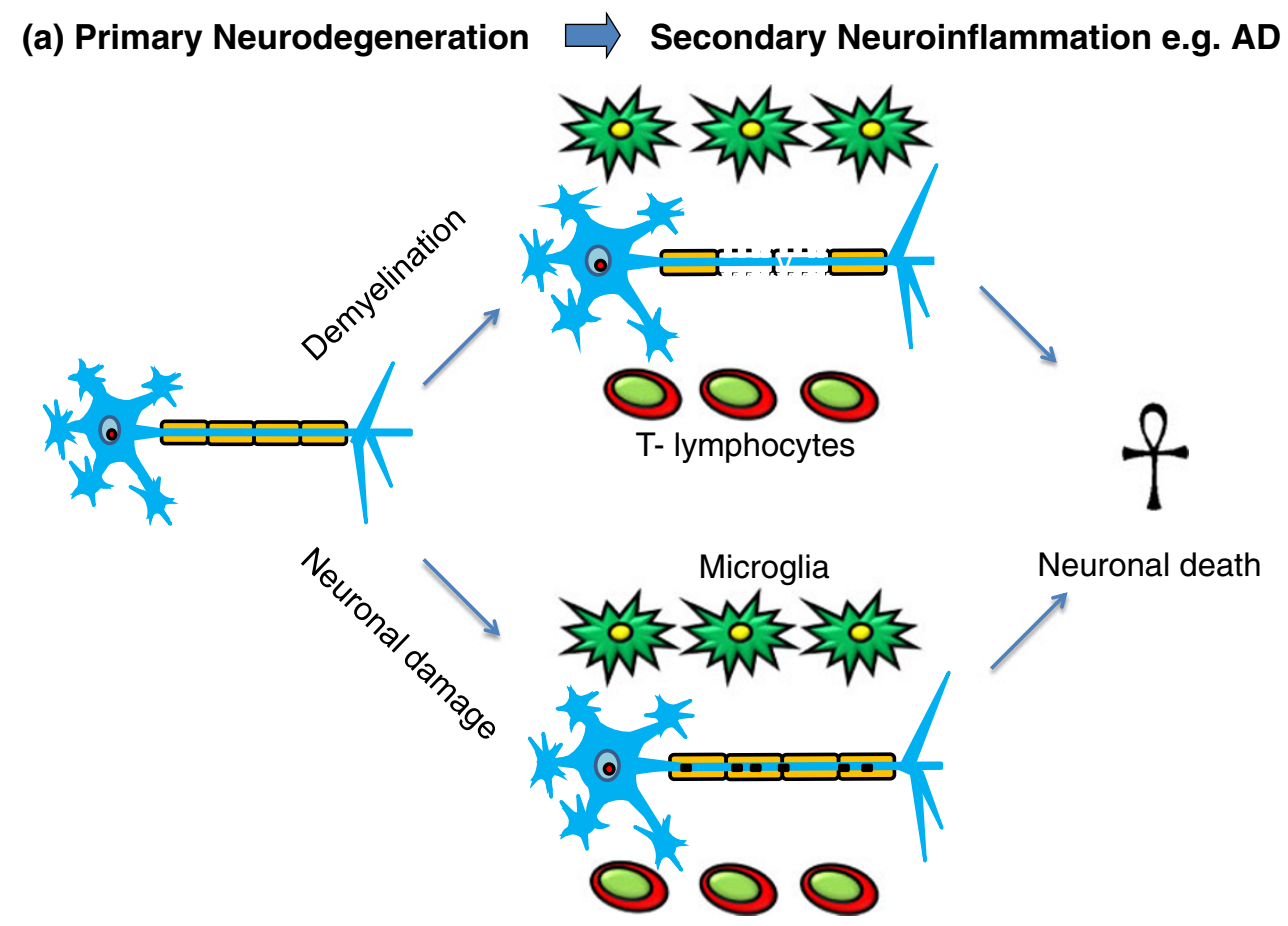

Neuronal death

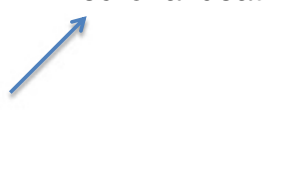

(b) Primary Neuroinflammation $\square$ Secondary Neurodegeneration e.g. MS

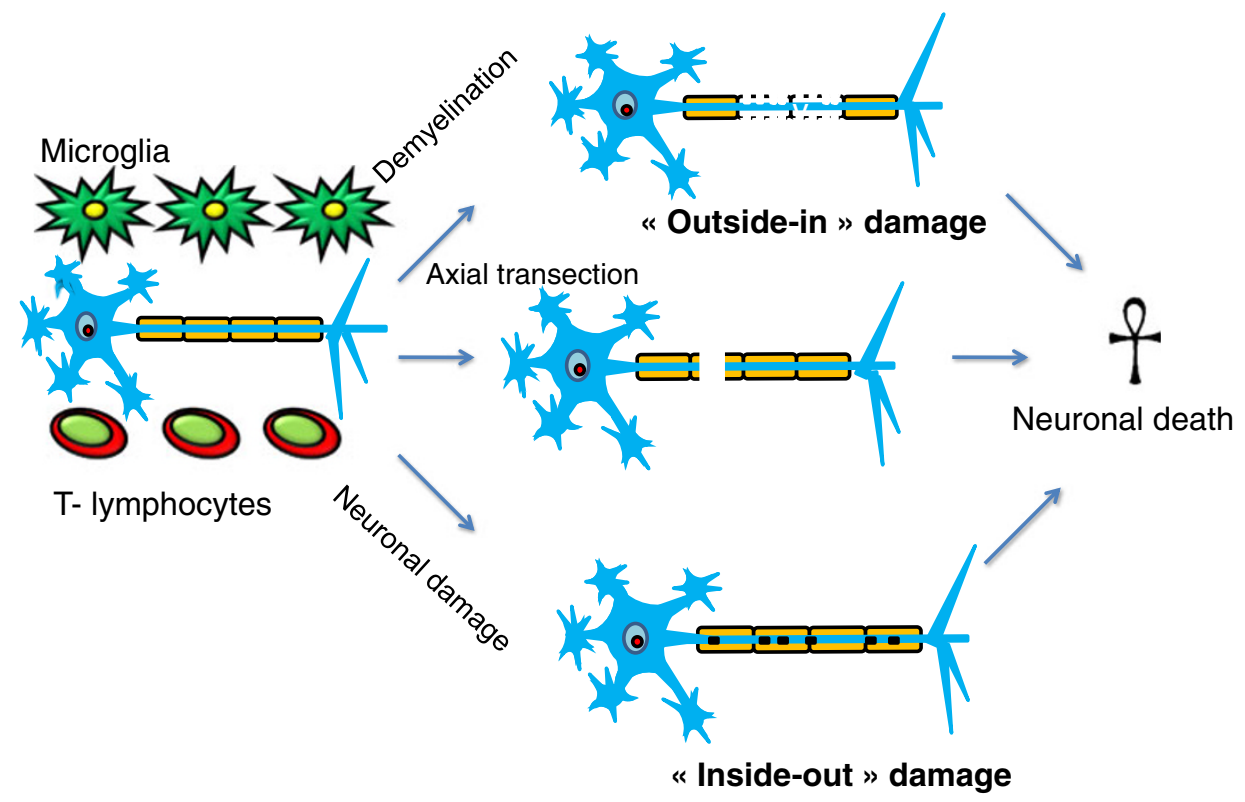

new insights into its structure and molecular functions. Although TSPO is found in many tissue types, it is expressed at the highest levels under normal conditions in tissues that synthesise steroids [13]. It has high level of expression in organs such as adrenals, kidneys, lungs and spleen [14]. The TSPO is also found in many other regions of the body, including human iris, ciliary body, heart, liver, testis, blood cells and brain. The TSPO is expressed at low levels primarily on microglia in healthy brain. It is expressed by the mitochondria of activated microglial cells in response to a variety of pathogens and insults [12]. TSPO can also be expressed in macrophage in inflammatory tissues associated with tumours [15]. It is significantly upregulated in breast, prostate, colon and brain cancers with protein expression linked to cancer progression and poor survival rates [13]. TSPO is a part of the mitochondrial 


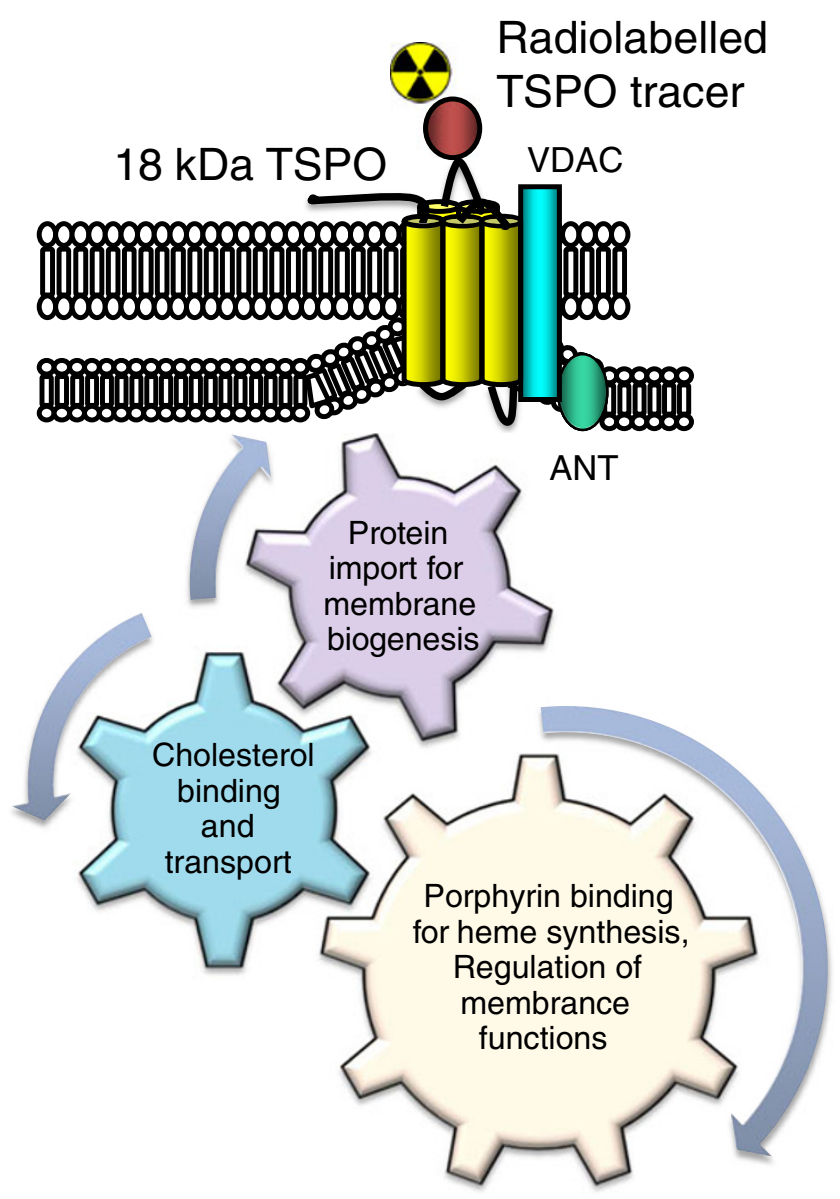

Fig. 4 TSPO structures and functions. It is a hetero-oligomeric complex and consists of at least three different subunits, including the isoquinolone binding protein of $18-\mathrm{kDa}$ TSPO, a voltage-dependent anion channel (VDAC) and an adenine nucleotide transporter (ANT). It contains inner and outer membrane contact sites to facilitate the passage of lipophilic molecules across the intermembrane space. The minimal functional unit or the binding site, however, is the $18-\mathrm{kDa}$ protein. TSPO has four main functions: (1) cholesterol binding and transport into mitochondria, a key function in steroidogenesis and bile salt synthesis, (2) protein import for membrane biogenesis, and other cellular functions including apoptosis, cell proliferation, and differentiation, (3) porphyrin binding and transport for heme synthesis, and (4) regulation of mitochondrial functions

permeability transition pore (MPTP). The MPTP consists of at least three different subunits on the mitochondrial outer membrane, including the isoquinolone binding protein of $18 \mathrm{kDa}$ (TSPO), a voltage-dependent anion channel of $32 \mathrm{kDa}(\mathrm{VDAC})$ and an adenine nucleotide transporter of $30 \mathrm{kDa}$ (ANT) [16] (Fig. 4). It contains inner and outer membrane contact sites to facilitate the passage of lipophilic molecules across the intermembrane space. The minimal functional unit or the binding site, however, is the $18-\mathrm{kDa}$ protein, which mainly consists of five transmembrane domains. Its importance does not depend only on ligand binding but also on the subsequent effects of binding
[8]. TSPO has four main functions: (1) cholesterol binding and transport into mitochondria, a key function in steroidogenesis and bile salt synthesis, (2) protein import for membrane biogenesis, and directly or indirectly for other cellular functions including apoptosis, cell proliferation, differentiation, and anion transport, (3) porphyrin binding and transport for heme synthesis, and (4) regulation of mitochondrial function and immune regulation [13, 16]. Although research suggests that there are multiple TSPO binding sites, the nature of these sites and their functional significance is still obscured [17]. It is not exactly known what the endogenous ligands of TSPO are but some propose they can be cholesterol, diazepam-binding inhibitor (DBI), a neuropeptide, or porphyrins [9]. It is possible that TSPO polymerisation is associated with steroidogensis and it results in a conformational change to the TSPO binding sites [17].

In the CNS, TSPO ligands have been found to stimulate synthesis of neurosteroids involved in diverse functions, from regulation of apoptosis to reduction of anxiety via modulation of $\mathrm{GABA}_{\mathrm{A}}$ receptor [16]. The TSPO expression is low in normal brain but it is upregulated in the brain at sites of injury and inflammatory tissues associated with tumour. The molecular mechanisms underlying these conditions are unknown, although some factors, such as reactive oxygen species, interleukin 1 and tumour necrosis factor (TNF) $\alpha$, may play a role. Local TSPO overexpression in response to damage makes it a potentially ideal and sensitive marker for the detection of subtle changes in the region of brain insult (Fig. 5).

\section{$\left[{ }^{11}\right.$ C]PK11195: the workhorse for TSPO imaging and its limitations}

PK11195 [1-(2-chlorophenyl)- $N$-methyl- $N$-(1-methylpropyl)-3-isoquinoline carboxamide] is the first nonbenzodiazepine and selective TSPO ligand labelled with carbon-11. It was discovered in 1984 and named by a French company, Pharmuka [18]. It remains most widely used in TSPO PET imaging with nanomolar binding affinity and it is the prototypical reference [19]. $\left[{ }^{11} \mathrm{C}\right]$ PK11195 has been used in many human CNS studies, including Rasmussen's encephalitis, MS, AD, PD, amyotrophic lateral sclerosis, HD, HIV, herpes encephalitis, and neuropsychiatric disorders such as schizophrenia [16]. Studies found increased binding of $\left[{ }^{11} \mathrm{C}\right] \mathrm{PK} 11195$ in corresponding MR regions of focal gadolinium-enhanced lesions and normal-appearing white matter (NAWM) in MS patients [20]. Brain atrophy, correlating with disease duration and disability of MS is directly related to $\left[{ }^{11} \mathrm{C}\right]$ PK11195 uptake, NAWM and inflammatory-related T2 hyperintense lesions [20, 21]. A study also showed an 


\section{TSPO Imaging Strategy}

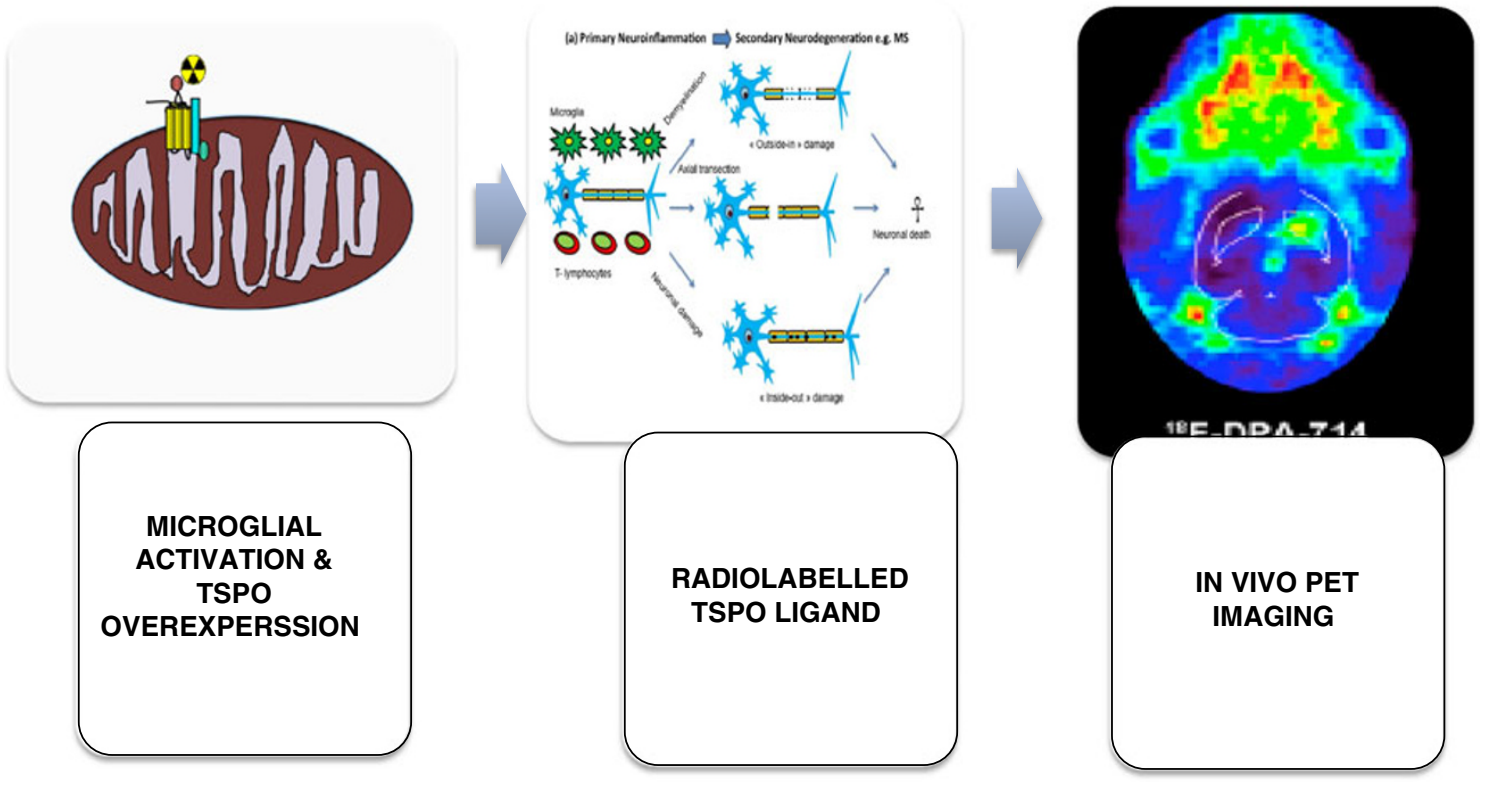

Fig. 5 In vivo TSPO imaging by PET. PET imaging of microglial cells in vivo involves utilising ligands labelled with ${ }^{11} \mathrm{C}$ or ${ }^{18} \mathrm{~F}$ radioisotopes that bind translocator protein (TSPO) expressed on the outer mitochon- drial membrane in microglia. The radioisotope undergoes positron emission decay underlying the principle of PET imaging

Although it is well established that $\left[{ }^{11} \mathrm{C}\right] \mathrm{PK} 11195$ shows increased uptake in a wide array of neurodegenerative disorders, it suffers from unresolved methodological and kinetic issues. There is low sensitivity and a limited capacity to quantify subtle TSPO expression in vivo. Relatively low receptor affinity could lead to low binding potential, which results from substantial binding of the tracer to other parts of the body. It suffers from high plasma protein binding and high non-specific binding likely related to high lipophilicity. The more lipophilic molecule is undesirable not only for slowing passive brain entry but also for increasing high non-specific binding to brain fats and protein. Relatively poor penetration of the blood brain barrier (BBB) and low brain uptake also lead to poor signal-to-noise ratio on PET imaging. Also, the lack of sensitivity and specificity and highly variable kinetic behaviour of $\left[{ }^{11} \mathrm{C}\right] \mathrm{PK} 11195$ have precluded the development of a standard quantitative method of analysis $[8,14]$. Without an on-site cyclotron facility, the short half-life of carbon-11 ( $\left.\mathrm{t}_{1 / 2}=20.38 \mathrm{~min}\right)$ also limits PET imaging using $\left[{ }^{11} \mathrm{C}\right] \mathrm{PK} 11195$ in routine clinical practice.

\section{Current trend of novel potential tracers}

In the light of the limitations of $\left[{ }^{11} \mathrm{C}\right] \mathrm{PK} 11195$, many groups worldwide are actively engaged in a search for new radiotracers with improved capacities to image and quantify 
Table 2 Examples of new potential PET tracers for TSPO imaging in clinical studies

\begin{tabular}{|c|c|c|c|}
\hline PET tracer & Chemical class & Diseased/healthy subjects & Findings \\
\hline$\left[{ }^{11} \mathrm{C}\right] \mathrm{DAA} 1106$ & Phenoarylacetamides [6] & $\mathrm{AD}, \mathrm{ALS}, \mathrm{FTD}, \mathrm{MS}, \mathrm{CI}$ & $\begin{array}{l}\text { Higher binding affinity compared } \\
\text { with PK11195 }[33,43]^{\mathrm{a}}\end{array}$ \\
\hline$\left[{ }^{11} \mathrm{C}\right]$ vinpocetine & Vina alkaloids[4] & MS, healthy subjects & $\begin{array}{l}\text { Higher BP but potential different binding } \\
\text { sites as PK11195 [44-46] }\end{array}$ \\
\hline$\left[{ }^{18} \mathrm{~F}\right] \mathrm{FEDAA} 1106$ & Phenoarylacetamides [6] & Healthy subjects & Match known patterns of TSPO distribution $[47]^{\mathrm{a}}$ \\
\hline$\left[{ }^{11} \mathrm{C}\right] \mathrm{PBR} 28$ & Phenoarylacetamides [6] & Healthy subjects & Good agreement of pattern in TSPO distribution [35] $]^{\mathrm{a}}$ \\
\hline$\left[{ }^{11} \mathrm{C}\right] \mathrm{AC}-5216$ & Oxodihydropurines [5] & Healthy subjects & Match known patterns of TSPO distribution $[36]^{\mathrm{a}}$ \\
\hline$\left[{ }^{11} \mathrm{C}\right] \mathrm{DPA}-713$ & Pyrazolo-[1,5-a]-pyrimidines [7] & Healthy subjects & Potentially higher binding affinity than PK11195 [37 ${ }^{\mathrm{a}}$ \\
\hline$\left[{ }^{18} \mathrm{~F}\right] \mathrm{DPA}-714$ & Pyrazolo-[1,5-a]-pyrimidines [7] & Healthy subjects & Potentially higher binding potential in brain [38] \\
\hline
\end{tabular}

$A D$ Alzheimer's disease, $A L S$ amyotrophic lateral sclerosis, $M S$ multiple sclerosis, FTD frontotemporal dementia, $C I$ cerebral infarction

${ }^{a}$ Mixed-affinity binding in human brain

TSPO expression. In the literature, a number of excellent comprehensive reviews have been published on TSPO imaging [8, 9, 14, 16, 28]. Schweitzer et al. [16] and Rupprecht et al. [9] offer more details on the updated trend of TSPO radiotracers development.

TSPO radiotracers can be basically subdivided into seven chemical classes. Extensive research during recent years has led to the development of more than 50 new TSPO tracers $[8,14,16,29]$. New tracers that used in human studies include $\left[{ }^{11} \mathrm{C}\right] \mathrm{DAA} 1106,\left[{ }^{18} \mathrm{~F}\right]$ FEDAA1106, $\left[{ }^{11} \mathrm{C}\right]$ PBR 28, $\left[{ }^{11} \mathrm{C}\right]$ vinpocetine, $\left[{ }^{11} \mathrm{C}\right] \mathrm{AC}-5216,\left[{ }^{11} \mathrm{C}\right]$ DPA713 and $\left[{ }^{18}\right.$ F]DPA714 [30-38] (Table 2). The studies in humans are at their initial stages with sporadic findings. From the glimpse of information, $\left[{ }^{11} \mathrm{C}\right] \mathrm{DAA} 1106,\left[{ }^{18} \mathrm{~F}\right]$ FEDAA1106, and $\left[{ }^{11} \mathrm{C}\right] \mathrm{PBR} 28$ have shown significantly increased binding in living brain. The binding potential of $\left[{ }^{11} \mathrm{C}\right]$ vinpocetine is higher than $\left[{ }^{11} \mathrm{C}\right] \mathrm{PK} 11195$ in $\mathrm{MS}$ patients. $\left[{ }^{18} \mathrm{~F}\right] \mathrm{DPA}-714$, a close analogue of DPA-713, is also a promising PET tracer and has recently demonstrated high affinity for the TSPO with better uptake and binding potential than $\left[{ }^{11} \mathrm{C}\right] \mathrm{PK} 11195[19,38]$. More recent studies show $\left[{ }^{11} \mathrm{C}\right] \mathrm{SSR} 180575$, the first TSPO radioligand based on an indoleacetamide scaffold, has a better capacity than $\left[{ }^{11} \mathrm{C}\right] \mathrm{PK} 11195$ to distinguish inflammatory tissue from healthy brain tissue [39, 40]. Discrepancies are found between the results of the binding affinities of different new TSPO radiotracers, and they showed heterogeneity in binding potentials in humans [9, 16, 41, 42].

Most of the new TSPO tracers are, however, still in the infancy of investigation and some show mixed-affinity binding in human [41, 42]. Many research efforts in preclinical studies are aimed at the following key areas:
Fig. 6 Schematic representation of TSPO radiotracer in the brain shows the fates of TSPO radiotracer and its radiometabolites passing through the brain-blood barrier (BBB). Radiotracer design for TSPO for the brain aims to achieve optimal passive diffusion across the BBB without impediment by efflux transporters (E.T.) to give a high specific binding (indicated by red circles). Many research efforts are aimed at (1) radiotracer metabolic stability (no/ less radiometabolites), (2) high binding potential (high specific binding), (3) ligand-receptor kinetics (to optimise kinetic modelling), and (4) quantitative methods (Tradiotracer, $M$ radiometabolite, $f$ free, $b$ bound, $n b$ non-specific binding, $s b$ specific binding)

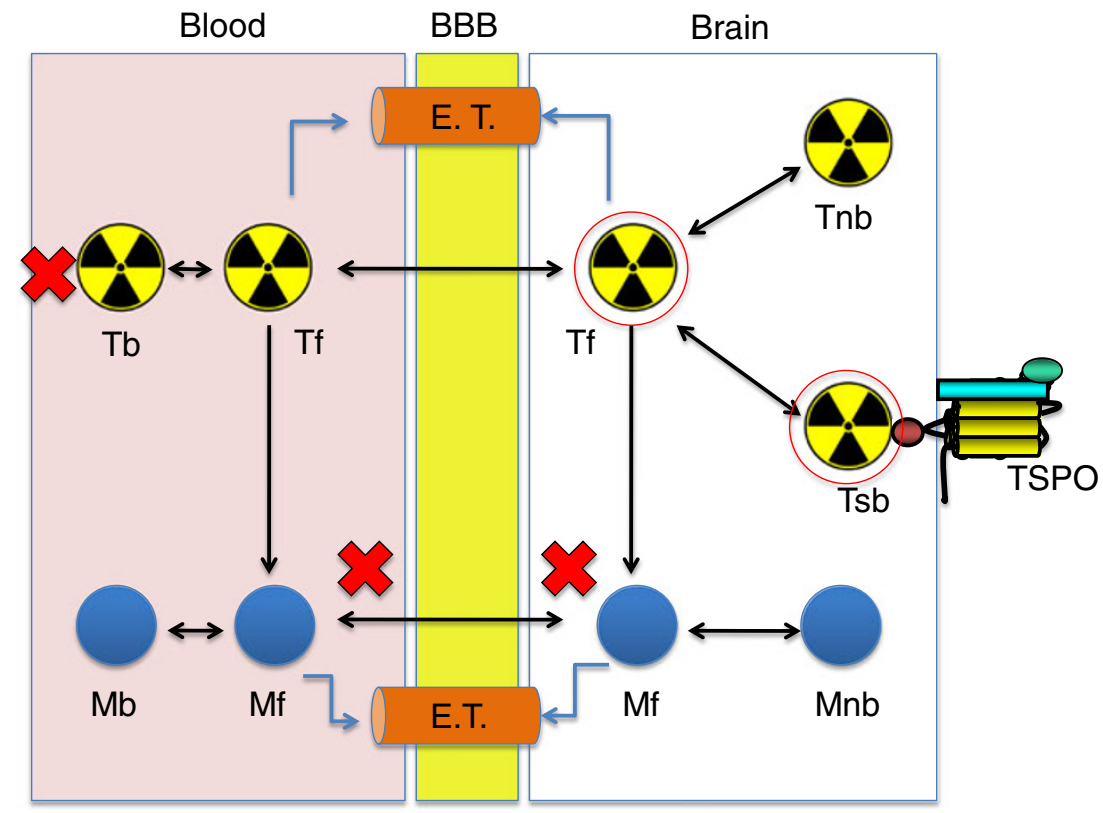


(1) ligand metabolic stability, (2) specific binding potential (BP), (3) ligand-receptor kinetics, and (4) quantitative methods. The new TSPO tracer must show high affinity and selectivity for the receptor. Ideally, a PET radiotracer should enter the brain and interact selectively with its target in the absence of troublesome radiometabolites. Practically, potential problems related to radiometabolites of the tracer must be limited through effective radiotracer design. It is essential for accurate in vivo measurement of the density of a target protein like TSPO. Binding potential measures, obtained with PET and a specific radiotracer, are considered to be proportional to the receptor density in vivo and high BP is crucial for TSPO quantification [5]. Suitable brain pharmacokinetics in relation to radiolabel half-life with observable brain uptake and washout kinetics is also desirable for measuring ligand-receptor interaction (Fig. 6).

\section{Perspectives}

There is accumulating evidence that neuroinflammation plays a crucial role in the development and progression of many neurodegenerative disorders such as MS, AD and HD. Collateral neuronal damage is clearly inherent to primary neuroinflammation, and neuroinflammation is a likely sequel of primary neurodegenerative diseases. It is important to understand the mechanisms that initiate neuroinflammation and neurodegeneration. Many questions remain regarding the use of TSPO tracers as diagnostic tools to assess activation of microglia. Overexpressed TSPO constitutes an important target for the detection or novel treatment of neuroinflammation and neurodegenerative disorders. The concept of multiple TSPO binding sites, variable conformational states of the protein and mixed binding affinity in living brain needs to be addressed when developing new PET TSPO radiotracers. Provided the broad spectrum of putative applications of TSPO ligands, further studies are eagerly awaited. With further development of potential tracers by better understanding of the protean nature of the receptor in healthy and diseased brain, PET imaging of the TSPO could offer a non-invasive modality for early diagnosis and theranostics of CNS diseases in ways beyond the scope of conventional imaging techniques.

Acknowledgments This work was supported by the FP6 European Networks of Excellence EMIL (LSHC-CT-2004-503569) and DIMI (LSHB-CT2005-5121146), and the STREP RATstream, (LSHM-CT2007-037846)

\section{References}

1. Weissleder R, Mahmood U (2001) Molecular imaging. Radiology 219:316-333
2. Pysz MA, Gambhir SS, Willmann JK (2010) Molecular imaging: current status and emerging strategies. Clin Radiol 65:500-516

3. Beyer T, Townsend DW, Czernin J, Freudenberg LS (2011) The future of hybrid imaging - part 2: PET/CT. Insights Imaging 2:225-234

4. Li Z, Conti PS (2010) Radiopharmaceutical chemistry for positron emission tomography. Adv Drug Deliv Rev 62:1031-1051

5. Pike VW (2009) PET radiotracers: crossing the blood-brain barrier and surviving metabolism. Trends Pharmacol Sci 30:431-440

6. Streit WJ, Mrak RE, Griffin WST (2004) Microglia and neuroinflammation: a pathological perspective. J Neuroinflammation 1:1-4

7. Zipp F, Aktas O (2006) The brain as a target of inflammation: common pathways link inflammatory and neurodegenerative diseases. Trends Neurosci 29:518-527

8. Doorduin J, de Vries EFJ, Dierckx RA, Klein HC (2008) PET imaging of the peripheral benzodiazepine receptor: monitoring disease progression and therapy response in neurodegenerative disorders. Curr Pharm Des 14:3297-3315

9. Rupprecht R, Papadopoulos V, Rammes G et al (2010) Translocator protein $(18 \mathrm{kDa})(\mathrm{TSPO})$ as a therapeutic target for neurological and psychiatric disorders. Nat Rev Drug Discov 9:971-988

10. De Stefano N, Narayanan S, Francis GS et al (2001) Evidence of axonal damage in the early stages of multiple sclerosis and its relevance to disability. Arch Neurol 58:65-70

11. Winkeler A, Boisgard R, Martin A, Tavitian B (2010) Radioisotopic Imaging of Neuroinflammation. J Nucl Med 51:1-4

12. Venneti S, Wiley CA, Kofler J (2009) Imaging microglial activation during neuroinflammation and Alzheimer's disease. J Neuroimmune Pharmacol 4:227-243

13. Batarseh A, Papadopoulos V (2010) Regulation of translocator protein $18 \mathrm{kDa}$ (TSPO) expression in health and disease states. Mol Cell Endocrinol 327:1-12

14. Chauveau F, Boutin H, Van Camp N, Dolle F, Tavitian B (2008) Nuclear imaging of neuroinflammation: a comprehensive review of [11C]PK11195 challengers. Eur J Nucl Mol Imaging 35:23042319

15. Roeda D, Kuhnast B, Damont A, Dolle F (2011) Synthesis of fluorine-18-labelled TSPO ligands for imaging neuroinflammation with positron emission tomography. J Fluorine Chem. doi:1016/j.fluchem.2011.03.020

16. Schweitzer PJ, Fallon BA, Mann JJ, Kumar JSD (2010) PET tracers for the peripheral benzodiazepine receptor and uses thereof. Drug Discov Today 15:933-942

17. Scarf AM, Kassiou M (2011) The translocator protein. J Nucl Med 52:667-680

18. Camsonne R, Crouzel C, Comar D et al (1984) Synthesis of N[11C]methyl, N-(methyl-1 propyl), (chloro-2 phenyl)-1 isoquinoleine carboxamide-3 (PK11195): a new ligand for peripheral benzodiazepine receptors. J Labelled Compds Radiopharm 21:985-991

19. Chauveau FC, Van Camp N, Dolle F et al (2009) Comparative evaluation of the translocator protein radioligands ${ }^{11} \mathrm{C}$-DPA-713, ${ }^{18} \mathrm{~F}-\mathrm{DPA}-714$, and ${ }^{11} \mathrm{C}-\mathrm{PK} 11195$ in a rat model of acute neuroinflammation. J Nucl Med 50:468-476

20. Versijpt J, Debruyne JC, Van Laere KJ et al (2005) Microglial imaging with positron emission tomography and atrophy measurements with magnetic resonance imaging in multiple sclerosis: a correlative study. Mult Scler 11:127-134

21. Debruyne JC, Versijpt J, Van Laere KJ et al (2003) PET visualization of microglia in multiple sclerosis patients using $\mathrm{C}$ 11 PK11195. Eur J Neurol 10:257-264

22. Thiel A, Radlinska BA, Paquette C et al (2010) The temporal dynamics of poststroke neuroinflammation: a longitudinal diffu- 
sion tensor imaging-guided PET study with C-11-PK11195 in acute subcortical stroke. J Nucl Med 51:1404-1412

23. Cagnin A, Kassiou M, Meikle SR, Banati RB (2006) In vivo evidence for microglial activation in neuro degenerative dementia. Acta Neurol Scand 114:107-114

24. Tai YF, Pavese N, Gerhard A et al (2007) Microglial activation in presymptomatic Huntington's disease gene carriers. Brain 130:1759-1766

25. Politis M, Pavese N, Tai YF et al (2011) Microglial activation in regions related to cognitive function predicts disease onset in Huntington's disease: a multimodal imaging study. Hum Brain Mapp 32:258-270

26. Doorduin J, de Vries EFJ, Willemsen ATM, de Groot JC, Dierckx RA, Klein HC (2009) Neuroinflammation in schizophrenia-related psychosis: a PET study. J Nucl Med 50:1801-1807

27. van Berckel BN, Bossong MG, Boellaard R et al (2008) Microglia activation in recent-onset schizophrenia: a quantitative (R)-C-11 PK11195 positron emission tomography study. Biol Psychiatry 64:820-822

28. Pimlott SL, Sutherland A (2011) Molecular tracers for the PET and SPECT imaging of disease. Chem Soc Rev 40:149-162

29. Dolle F, Luus C, Reynolds A, Kassiou M (2009) Radiolabelled molecules for imaging the translocator protein $(18 \mathrm{kDa})$ using positron emission tomography. Curr Med Chem 16:2899-2923

30. Damont A, Hinnen F, Kuhnast B et al (2008) Radiosynthesis of $\left[{ }^{18} \mathrm{~F}\right]$ DPA-714, a selective radioligand for imaging the translocator protein (18 kDa) with PET. J Labelled Compds Radiopharm 51:286-292

31. Boutin H, Chauveau F, Thominiaux C et al (2007) C-11-DPA-713: A novel peripheral benzodiazepine receptor PET ligand for in vivo imaging of neuroinflammation. J Nucl Med 48:573-581

32. James ML, Fulton RR, Vercoullie J et al (2008) DPA-714, a new translocator protein-specific ligand: Synthesis, radiofluorination, and pharmacologic characterization. J Nucl Med 49:814-822

33. Yasuno F, Ota M, Kosaka J et al (2008) Increased binding of peripheral benzodiazepine receptor in Alzheimer's disease measured by positron emission tomography with C-11 DAA1106. Biol Psychiatry 64:835-841

34. Fujimura Y, Zoghbi SS, Simeon FG et al (2009) Quantification of translocator protein $(18 \mathrm{kDa})$ in the human brain with PET and a novel radioligand, F-18-PBR06. J Nucl Med 50:1047-1053

35. Brown AK, Fujita M, Fujimura Y et al (2007) Radiation dosimetry and biodistribution in monkey and man of C-11-PBR28: a PET radioligand to image inflammation. J Nucl Med 48:2072-2079
36. Miyoshi M, Ito H, Arakawa R et al (2009) Quantitative analysis of peripheral benzodiazepine receptor in the human brain using PET with C-11-AC-5216. J Nucl Med 50:1095-1101

37. Endres CJ, Pomper MG, James M et al (2009) Initial evaluation of C-11-DPA-713, a novel TSPO PET ligand, in humans. J Nucl Med 50:1276-1282

38. Santiago-Ribeiro M, Vercouille J, Tauber C et al (2011) Cerebral kinetics and whold-body uptake of the TSPO radioligand $18 \mathrm{~F}$ DPA-714 in healthy volunteers. J Nucl Med 52:1279

39. Thominiaux C, Damont A, Kuhnast B et al (2010) Radiosynthesis of 7-chloro-N,N-dimethyl-5-[ $\left.{ }^{11} \mathrm{C}\right]$ methyl-4-oxo-3-phenyl-3,5-dihydro4H-pyridazino[4,5-b]indole-1-acetamide, $\left[{ }^{11} \mathrm{C}\right] \mathrm{SSR} 180575$, a novel radioligand for imaging the TSPO (peripheral benzodiazepine receptor) with PET. J Labelled Compds Radiopharm 53:767-773

40. Chauveau F, Boutin $\mathrm{H}$, Van Camp $\mathrm{N}$ et al (2011) In vivo imaging of neuroinflammation in the rodent brain with $\left[{ }^{11} \mathrm{C}\right] \mathrm{SSR} 180575$, a novel indoleacetamide radioligand of the translocator protein (18 kDa). Eur J Nucl Med Mol Imaging 38:509-514

41. Owen DR, Howell OW, Tang SP et al (2010) Two binding sites for H-3 PBR28 in human brain: implications for TSPO PET imaging of neuroinflammation. J Cereb Blood Flow Metab 30:1608-1618

42. Owen DRJ, Gunn RN, Rabiner EA et al (2011) Mixed-affinity binding in humans with $18-\mathrm{kDa}$ translocator protein ligands. J Nucl Med 52:24-32

43. Venneti S, Wang GJ, Nguyen J, Wiley CA (2008) The positron emission tomography ligand DAA1106 binds with high affinity to activated microglia in human neurological disorders. J Neuropathol Exp Neurol 67:1001-1010

44. Gulyas B, Halldin C, Sandell J et al (2002) PET studies on the brain uptake and regional distribution of $[11 \mathrm{C}]$ vinpocetine in human subjects. Acta Neurol Scand 106:325-332

45. Gulyas B, Halldin C, Sovago J et al (2002) Drug distribution in man: a positron emission tomography study after oral adminstration of the labelled neuroprotective drug vinpocetine. Eur J Nucl Med Mol Imaging 29:1031-1038

46. Vas A, Sovago J, Halldin C et al (2002) Cerebral uptake and regional distribution of $[11 \mathrm{C}]$-vinpocetin after intravenous adminstration to healthy men: a PET study. Orv Hetil 143:26312636

47. Fujimura Y, Ikoma Y, Yasuno F et al (2006) Quantitative analyses of F-18-FEDAA 1106 binding to peripheral benzodiazepine receptors in living human brain. J Nucl Med 47:43-50 